\title{
Results of Four-Level Anterior Cervical Discectomy and Fusion Using Stand-Alone Interbody Titanium Cages
}

\author{
Mohamed Alhashash ${ }^{1,2}$, Hassan Allouch $^{1}$, Heinrich Boehm ${ }^{1}$, Mootaz Shousha ${ }^{1,2}$ \\ ${ }^{I}$ Spine Surgery Department, Zentralklinik Bad Berka, Bad Berka, Germany \\ ${ }^{2}$ Orthopaedic and Trauma Department, Alexandria University, Alexandria, Egypt
}

Study Design: This is a retrospective study with a minimum follow-up of 2 years.

Purpose: The aim of this study is to assess the long-term outcomes after performing the four-level anterior cervical discectomy and fusion (ACDF) in the treatment of degenerative cervical spine disease using stand-alone titanium cages.

Overview of Literature: Over the last decades, a rapid increase in the use of stand-alone cages for ACDF has been observed. However, research on their application in the treatment of four-level diseases is insufficient.

Methods: In this study, 130 patients presenting with symptomatic cervical spondylosis who underwent four-level ACDF using standalone cages in our institution between 2008 and 2016 were assessed. Fifty-two patients were women and 78 men with a mean age of 60.5 years. Their clinical and radiological outcomes were assessed. The results of the Neck Disability Index (NDI) and Visual Ana$\log$ Scale as well as bony fusion were evaluated, and the revisions were analyzed. All of the patients underwent the four-level microscopic ACDF using the same titanium rectangular cage.

Results: The mean follow-up was $47 \pm 11.4$ months. A fusion of all four levels was achieved in $80.72 \%$ of the patients. In 25 patients $(19.23 \%)$, an incomplete bony bridging was observed in at least one fusion level at the final follow-up. However, only two patients $(1.5 \%)$ were symptomatic and underwent revision. The mean NDI improved significantly from $39.4 \pm 9.3$ at presentation to $8.3 \pm 6.6$ at the final follow-up. Cervical lordosis improved significantly from a mean of $5.5^{\circ}$ preoperatively to a mean of $15^{\circ}$ postoperatively. Cage sinking and loss of segment height during healing had a mean of $3 \mathrm{~mm}$.

Conclusions: Overall, the application of four-level ACDF using titanium cages in a stand-alone technique has been proven to be a safe and effective treatment method for degenerative disease. In a large cohort, a high rate of good long-term clinical and radiological results was achieved.

Keywords: Cervical vertebrae; Cages; Cervical; Fusion; 4-Levels; Spondylosis; Stand-alone

Received Sep 3, 2020; Revised Oct 17, 2020; Accepted Oct 19, 2020

Corresponding author: Mohamed Alhashash

Spine Surgery Department, Zentralklinik Bad Berka, Robert-Koch-Allee 7, 99437 Bad Berka, Germany

Tel: +49-36458541417, Fax: +49-3645853517, E-mail: mohamed.alhashash@zentralklinik.de 


\section{Introduction}

Multi-level disc disease of the cervical spine represents a challenging problem. Although a variety of anterior, posterior, and combined approaches have been advocated for multi-level cervical disc disease, the anterior approach still represents the preferable surgery in many cases as it allows for the direct decompression of the spinal cord and nerve roots as well as achieving solid fusion [1]. Multilevel cervical discectomy is often combined with anterior or posterior instrumentation to maintain the spinal curvature and increase the fusion rate [2]. However, instrumentation has additional hazards, such as implant-related complications, increased operative time, and blood loss $[2,3]$.

The posterior decompression for multi-level cervical spinal canal stenosis is effective [4]. Studies showed good results in cases with preserved cervical lordosis or in cases of ossification of the posterior longitudinal ligament as the anterior approach in these cases is associated with an increased rate of dural injury and hematoma [5]. On the other hand, the posterior approach had significantly more wound healing problems, and the correction of cervical lordosis is not as significant as the anterior approach $[4,5]$.

The use of the anterior cervical plates reduces the graft dislocations and improves cervical lordosis after anterior decompression and fusion [6]. The modern cages with built-in lordosis and the cage design that allows a better press-fit insertion of the cages provide stability even without an additional plate fixation [7]. Zero-profile implants reduce plate-associated complications such as dysphagia and loosening of the screws $[1,2]$.

Recently, a rapid expansion in the use of cervical spine interbody fusion cages [2]. Anterior cervical discectomy and fusion (ACDF) is increasingly performed using standalone cages, which overcomes the limitations associated with additional instrumentation [3]. Recent publications have assessed the safety and efficacy of stand-alone cages in short-segment cervical spinal fusion $[8,9]$. Because the documented literature for four-level ACDF is limited, the aim of this study is to assess the long-term clinical and radiological outcomes after performing four-level ACDF in the treatment of degenerative cervical spine disease with segmental stenosis using anterior stand-alone titanium cages without additional instrumentation.

\section{Materials and Methods}

\section{Patients}

A retrospective review of the prospectively collected database for patients undergoing a primary four-level ACDF between 2008 and 2016 in the spine surgery department, Zentralklinik Bad Berka, Germany was conducted. The indication for surgery in these patients was a degenerative disease with spinal canal stenosis extending from $\mathrm{C} 3$ to C7 diagnosed using a preoperative magnetic resonance imaging (MRI) and presenting with persistent cervical radiculopathy or cervical myelopathy despite 6 weeks of conservative treatment. In the case of radiculopathy presenting with a motor deficit, operative treatment was done without delay. Patients presenting with newly diagnosed cervical myelopathy or a recent progression of preexisting myelopathy were immediately operated without preoperative conservative treatment. An independent neurologist examined each patient in this work, and the decision for surgery was taken after consultation of the neurology and radiology departments.

\section{Indications of surgery}

The Zentralklinik Bad Berka is a tertiary referral hospital with an average of 2,000 spine surgeries yearly. During the study period, ACDF was performed in 2,700 patients, and only 165 patients (6\%) underwent four-level ACDF. After consultation between the three departments (the neurology, radiology, and spine surgery departments), only patients with neurological and radiological signs were subjected to a four-level ACDF.

The neurologist examined the presence and severity of cervical myelopathy and/or cervical radiculopathy. The neuroradiologist provided the radiological statement regarding the affected levels (absolute spinal canal stenosis, neuroforamen stenosis, or radiological signs of myelopathy). In some patients, no clinical signs of cervical myelopathy were found despite the presence of radiological signs of myelopathy in the preoperative MRI examinations, even in levels that did not present with clinical signs of radiculopathy. In other patients, clinical signs of radiculopathy were observed in one or more levels, but radiologically, absolute spinal canal stenosis and/or neuroforamen stenosis extending in all the four levels was observed. The decision to perform the four-level surgery 
was made on the basis of both the clinical and radiological signs.

The exclusion criteria for the analysis were as follows: (1) patients with a history of rheumatoid arthritis or previous cervical spine surgery, (2) patients who underwent corpectomy at any of the intervening levels, and (3) patients with spondylodiscitis and post-traumatic cervical spine instability.

The search in the surgical database revealed a total of 164 patients fulfilling these criteria. In these patients, $\mathrm{ACDF}$ was performed at levels $\mathrm{C} 3 / 4$ to $\mathrm{C} 6 / \mathrm{C} 7$ using a titanium rectangular cage. Among the 164 operated patients, 34 were excluded because they did not continue with the minimum follow-up of 2 years-most of them being international patients not available for evaluation at the time of data collection. The remaining 130 patients were followed up for a minimum of 2 years and were all included in this study. Ethics approval was obtained from the Institutional Review Board of Alexandria University (IRB approval no., 128-2018) before the study was conducted. Informed consent was obtained from all individual participants included in the study.

\section{Surgical technique}

ACDF was performed using the standard Smith-Robinson approach [10]. A single transverse skin incision at the C5 level was performed, and the platysma muscle was split longitudinally in the direction of its fibers. The carotid vessels are prepared laterally. The strap muscles were prepared to access the anterior aspect of the cervical spine. After adequate decompression, a rectangular titanium cage was placed in the distracted intervertebral space under microscopic visualization of the dura, facilitated by the open design of the implant. After the verification of the correct position via fluoroscopy, the endplate areas that were not in contact with the footprint of the cage, thus were not weight bearing, were decorticated through the openly designed implant to provide an optimal bed for fusion. After that, all cages were filled with cylinders of autogenous iliac bone graft harvested in a minimally invasive technique with the aid of a special drill-trocar instrument. A local closed negative suction drain was inserted and removed after 48 hours. Postoperatively, a Philadelphia cervical collar was applied for 3-4 weeks.

\section{Outcomes assessment}

The preoperative, intraoperative, and postoperative data were collected. All X-rays were taken, stored, and measured in digital form. The intraoperative profile assessment included operative time, blood loss, and intraoperative complications. The clinical and radiographic followup was maintained for a mean period of $47 \pm 11.4$ months, with a minimum of 24 months. The clinical outcomes were determined on the basis of the results of the Neck Disability Index (NDI), which is a subjective pain questionnaire, and the Visual Analog Scale (VAS). The questionnaires were completed immediately before surgery and at the final follow-up. Plain cervical spine radiographs were obtained on the first and fifth postoperative days before discharge, followed by a regular evaluation over the follow-up period. A regular follow-up in the outpatient clinic was planned. The first visit is after 3 months, the second after 6 months, and the third after 1 year. The patients were requested to undergo an X-ray examination and clinical follow-up yearly. Any patient with new symptoms can contact our department for a follow-up visit. Postoperative dysphagia was assessed using the Bazaz Dysphagia Score directly after the operation and during the follow-up period [11]. Bazaz et al. [11] classified dysphagia according to severity and if dysphagia is present with liquids or solid intake. Any graft-related complications were recorded.

Radiographic fusion was determined according to the anteroposterior and dynamic lateral cervical spine X-rays in flexion and extension (Fig. 1). Fusion was defined as the presence of trabecular bone across the interfaces without lucencies between the cage and vertebral endplates and bony bridging formation between the superior and inferior endplates and the presence of $<1 \mathrm{~mm}$ of motion in the operated segment in dynamic X-ray views [12]. Two independent radiologists performed the radiological analysis. The results of the radiological evaluation were assessed by the authors. Fusion was considered only after the confirmation of both radiologists. If one or both radiologists did not confirm the fusion, the case was considered a nonfusion. Furthermore, the cervical lordosis angle between $\mathrm{C} 2$ and $\mathrm{C} 7$ and the height of the fused segment $\mathrm{C} 3-7$ were measured and compared over the follow-up period. Any reoperation during the follow-up period was reported and analyzed. 
Table 1. Changes in the clinical and radiological parameters before and after the operative treatment and the causes of revisions

\begin{tabular}{|c|c|c|c|}
\hline Variable & Preoperative & At the final follow-up & Significance (p-value $)^{a)}$ \\
\hline VAS neck pain & $8.4 \pm 1.5$ & $2.95 \pm 1.5$ & 0.01 \\
\hline VAS arm pain & $7.8 \pm 1.8$ & $2.3 \pm 2.0$ & 0.00 \\
\hline Neck Disability Index & $39.4 \pm 9.3$ & $8.3 \pm 6.6$ & 0.02 \\
\hline Cervical lordosis $\left({ }^{\circ}\right)$ & $5.5 \pm 13.5(-15$ to 10$)$ & $10 \pm 7.4$ (5 to 15$)$ & 0.031 \\
\hline Segment height (mm) & $88 \pm 4.2$ & $94 \pm 3.2$ & 0.07 \\
\hline Clinical motor radiculopathy & 85 & - & \\
\hline Clinical myelopathy & 45 & 2 & \\
\hline \multicolumn{4}{|l|}{ Reoperations } \\
\hline Postoperative hematomas & 4 & & \\
\hline Residual stenosis & 2 & & \\
\hline Symptomatic pseudoarthrosis & 2 & & \\
\hline Cage subsidence & 1 & & \\
\hline
\end{tabular}

Values are presented as mean $\pm S D$, mean $\pm S D$ (range), or number or patients.

VAS, Visual Analogue Scale; SD, standard deviation.

${ }^{a} B y$ Student $t$-test.

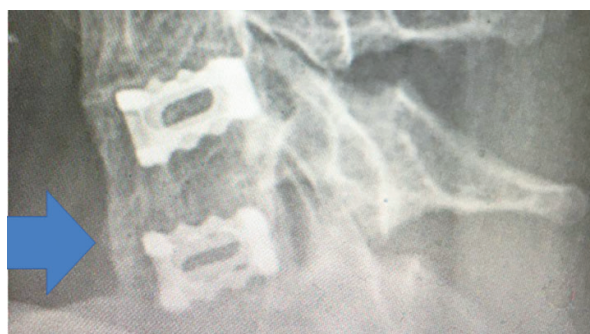

(A)

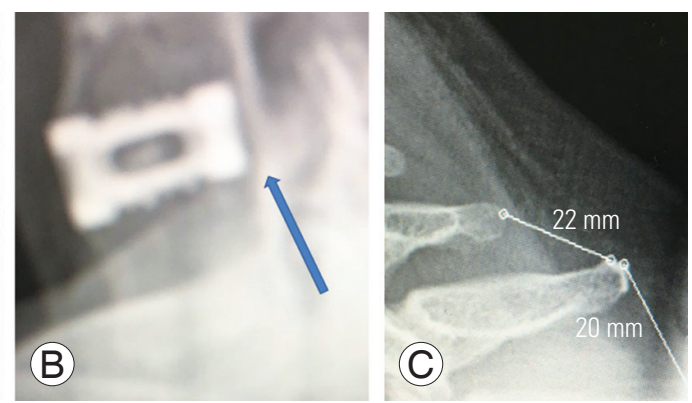

Fig. 1. Radiological fusion depended on the presence of bridging bone anterior to the cage ( $\mathbf{A}$, the broad arrow) or posterior to the cage (B, the little arrow). The distance between the spinous process tip was measured after the digital magnification of the $X$-rays to allow accurate measurement in this case $22 \mathrm{~mm}$ in flexsion and $20 \mathrm{~mm}$ in extension (C).

\section{Statistical analysis}

The collected data were analyzed using the IBM SPSS ver. 19.0 (IBM Corp., Armonk, NY, USA). Demographic data and frequencies were calculated using the mean and standard deviation. Clinical and radiological parameters were analyzed using paired $t$-test. Statistical significance was established at a $p$-value of $<0.05$.

\section{Results}

\section{Perioperative data}

Among the 130 patients, 52 were women (40\%) and 78 were men $(60 \%)$ (Table 1$)$. The mean age at surgery was $60.5 \pm 10.54$ years. The mean operative time was $196 \pm 46$ minutes, and the mean amount of blood loss was $500 \pm 380$ $\mathrm{mL}$. No intraoperative complications were reported. In the early postoperative phase and before discharge, seven patients had to be re-operated. The causes of these early reoperations were postoperative hematoma in four patients $(3.1 \%)$ (three with retropharyngeal hematoma and one with epidural hematoma). The clinical presentation was progressive postoperative dysphagia. An MRI was performed, and revisions were performed in case of mechanical compression of the esophagus due to the hematomas. In another two patients with dysphagia without significant MRI signs of mechanical esophageal compression, conservative therapy was applied with corticosteroids and a modification of the meals with specialized training for swallowing by logopedic specialists. Residual neuroforamen stenosis occurred in two patients (1.5\%) 

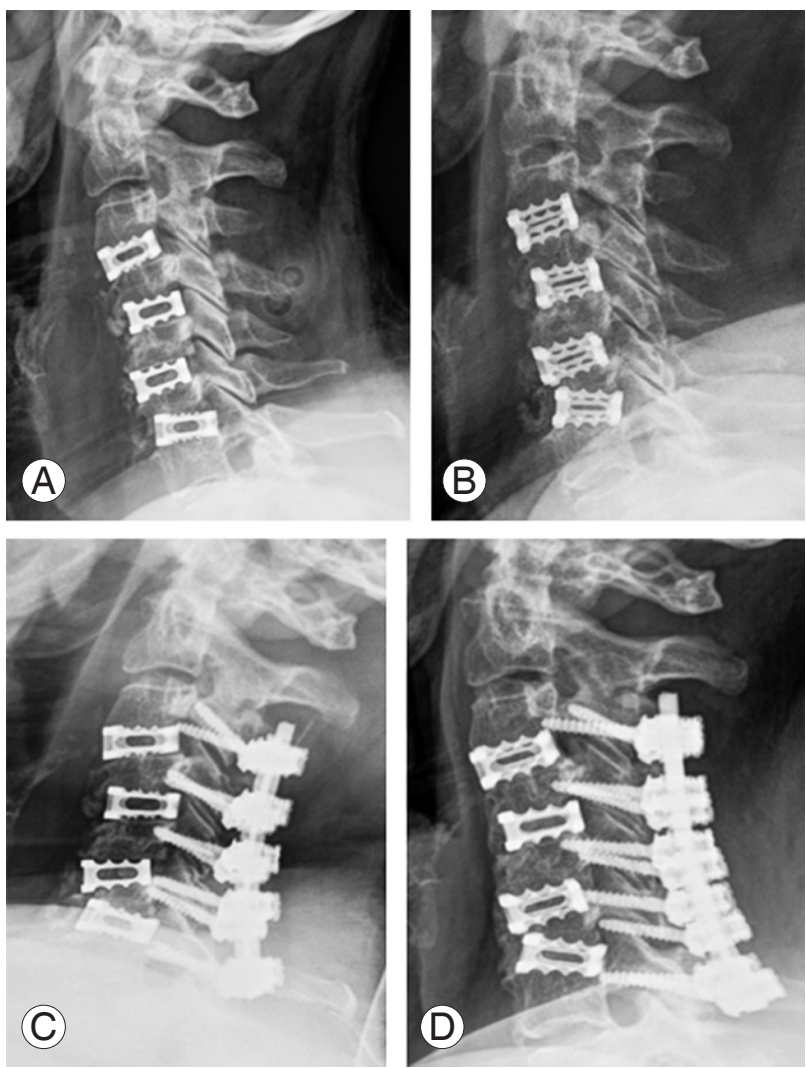

Fig. 2. The single case with postoperative cage subsidence necessitating revision before discharge. Plain X-ray directly after the first surgery $(\mathrm{A})$ and 10 days postoperative (B). (C) The case was treated by posterior instrumentation to avoid further deterioration of the sagittal profile, and (D) complete fusion at the final follow-up was achieved.

in which both had a postoperative motor weakness; a postoperative MRI and computed tomography (CT) were performed and showed significant neuroforamen stenosis. The revision was through the anterior removal of the implant in the affected level, decompression of the foramen, and reimplantation of the cages. Cage subsidence with kyphosis occurred in only one patient $(0.8 \%)$. In this single patient, posterior stabilization was added before discharge (Fig. 2).

\section{Clinical outcomes}

The mean value for the NDI improved significantly from $39.4 \pm 9.3$ at presentation to $8.3 \pm 6.6$ at the final followup ( $p=0.02$ and $t=2.35)$. The mean VAS for neck pain improved significantly from $8.4 \pm 2.95$ at presentation to $2.95 \pm 1.5$ at the final follow-up ( $p=0.01$ and $t=3.4$ ). Similarly, the mean VAS for arm pain improved from $7.8 \pm 1.8$ preoperatively to $2.3 \pm 2$ at the final follow-up ( $p=0.00$ and $t=2.23)$. Statistically, a significant improvement in symptoms was observed in all patients. At the end of the follow-up, no residual motor radiculopathy was found. Only two patients still presented with myelopathy despite adequate decompression. The two patients had the symptoms for a long time before the surgical decompression (5 and 11 months).

Regarding the complications, three patients (2.3\%) developed C5 palsy postoperatively on one side. This was manifested by deltoid weakness grade 2 , together with biceps weakness grade 4 . Full recovery of the muscle power was reported 6 months postoperatively in all three patients without revision. Another three patients (2.3\%) developed dysphagia due to retropharyngeal hematoma. All three patients were revised with the evacuation of the hematoma and immediate improvement of the dysphagia. Residual dysphagia was not reported in any of the 130 patients (Using Bazaz score) [7]. Two patients had numbness and radicular pain and weakness corresponding to C6 dermatome due to the residual stenosis of the neuroforamen C5/6 and underwent a revision with the decompression of the neuroforamen. Transient paraparesis occurred in one patient $(0.8 \%)$ in the early hours after surgery. An epidural hematoma was diagnosed using urgent postoperative MRI, and the evacuation of the hematoma was performed with complete resolution of the neurological symptoms before discharge. Recurrent laryngeal nerve dysfunction with hoarseness of voice occurred in one patient $(0.8 \%)$ and was managed conservatively using corticosteroid with satisfactory resolution over the follow-up period. Ten patients (7.7\%) had a transient iliac graft site pain without any residual pain after 3 months.

\section{Radiological outcomes}

At the last follow-up examination, 105 patients (80.77\%) had a radiographic fusion at all four levels, as demonstrated by bridging the bone between the vertebral bodies or the absence of $>1$-mm motion on dynamic X-rays (Fig. $3)$. In the remaining 25 patients (19.23\%), at least one level did not fulfill the criteria for solid bony fusion (Fig. 4). However, in most of these patients, no revision surgery was necessary because of the lack of symptoms necessitating surgical intervention. A CT scan was performed in case of failure to demonstrate the fusion in the X-rays (including the lateral functional views). CT was performed in 25 cases. Cases with the absence of trabecular fusion in 

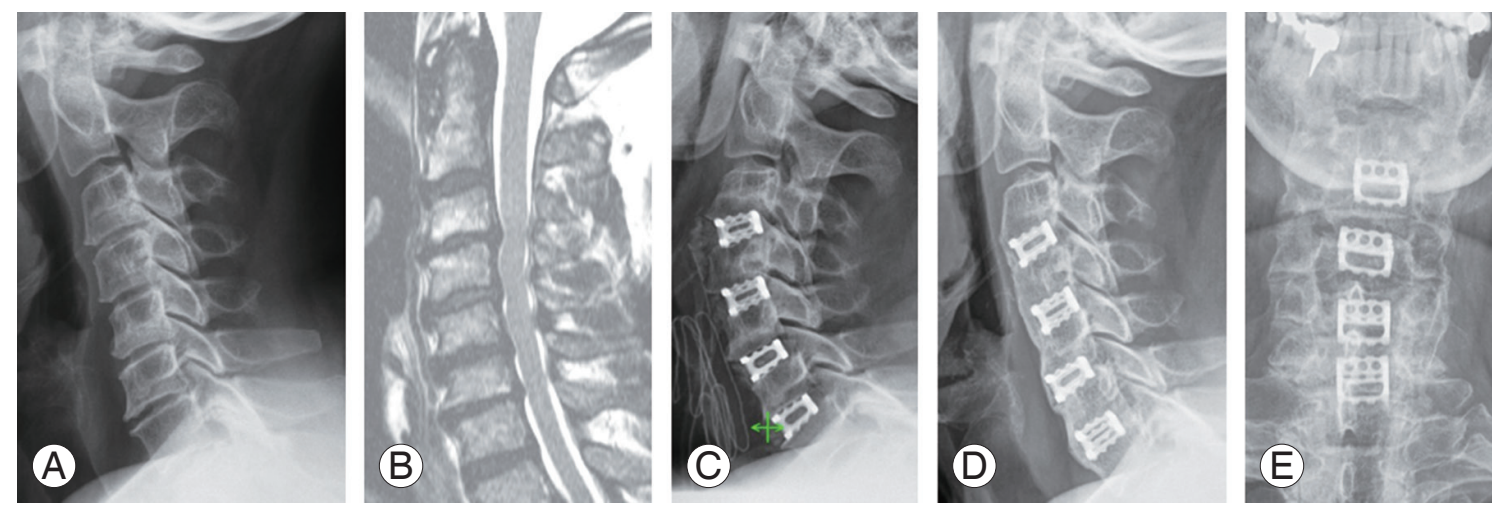

Fig. 3. A 65-year female patient: (A) preoperative lateral plain radiography, (B) preoperative magnetic resonance imaging showing stenosis C3-7, (C) lateral X-ray picture directly postoperatively, and (D, E) lateral and anteroposterior X-rays 2 years postoperatively showing solid fusion of the arthrodesis and proper cervical lordosis.
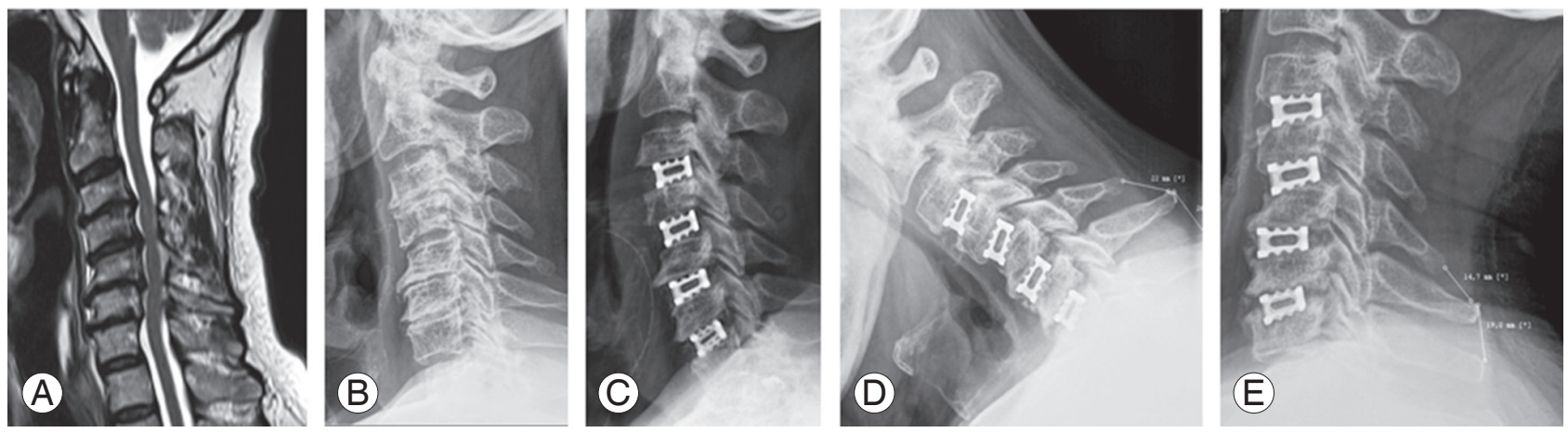

Fig. 4. An example of a case with asymptomatic pseudoarthrosis. (A) Preoperative magnetic resonance imaging showing stenosis C3-7, (B) preoperative lateral plain radiography, (C) lateral X-ray picture directly postoperatively, $28 \mathrm{~mm}$ in flexion (D) and $25 \mathrm{~mm}$ in extension (E) X-rays 2 years postoperatively showing absent bony bridging and more than 1-mm motion between the spinous processes. However, the patient was asymptomatic with no need for revision surgery.
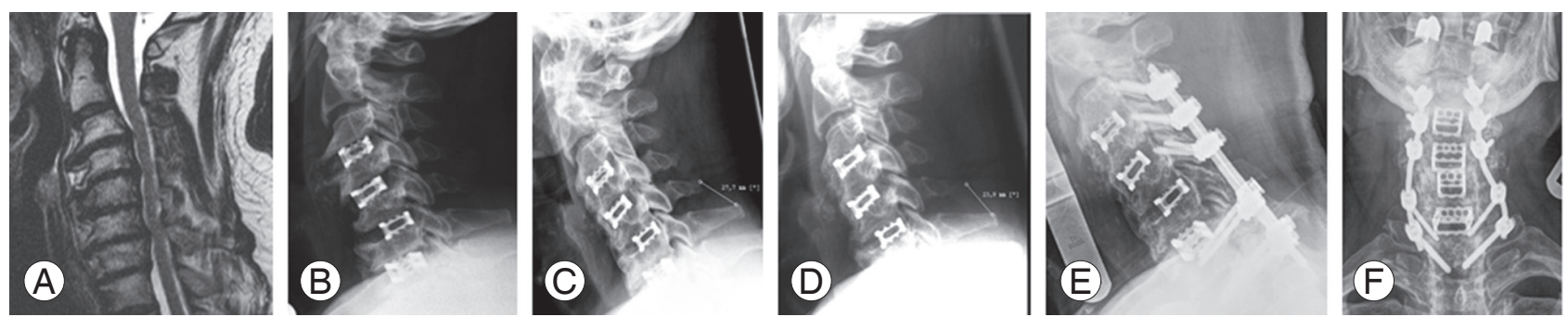

Fig. 5. One of the two cases with symptomatic pseudoarthrosis. (A) Preoperative magnetic resonance imaging showing stenosis C3-7, (B) lateral X-ray picture directly postoperatively, $27 \mathrm{~mm}$ in flexion (C) and $24 \mathrm{~mm}$ in extension (D) X-rays 28 months postoperatively showing absent bony bridging and more than 1 -mm motion between the spinous processes of $\mathrm{C} 5 / 6$, presenting with persistent axial neck pain. (E, F) Final lateral and anteroposterior X-ray pictures after posterior stabilization and fusion.

CT or the presence of lucency in the cage-bone interface were considered radiological non-fusion.

Two patients (1.5\%) suffered from axial neck pain due to pseudarthrosis and were revised after 28 and 36 months. The revision was performed in the form of posterior instrumentation using lateral mass screws and dorsal fusion (Fig. 5). The axial neck symptoms were relieved in both patients at the final follow-up with an NDI of 8 and 12 , respectively.

Regarding the cervical lordosis angle, a mean value of $5.5^{\circ}$ was reported preoperatively, ranging from $10^{\circ}$ kyphosis to $15^{\circ}$ lordosis. The mean postoperative cervical lordosis was $15^{\circ}$ and that at the final follow-up examination was $10^{\circ}$. Despite a mean loss of $5^{\circ}$ in cervical lordosis over the 
follow-up period, the mean final improvement was statistically significant ( $p=0.031$ and $t=2.67$ ) compared with the mean preoperative value. Similarly, the height of the fused segment improved markedly from a mean of $88 \mathrm{~mm}$ preoperatively to a mean of $94 \mathrm{~mm}$ postoperatively. At the final follow-up, a mean loss in the segmental height of $3 \mathrm{~mm}$ was reported, but without a statistical significance ( $p=0.07$ and $t=3.25$ ). Cage subsidence was assessed by measuring the fused segment height in the postoperative lateral X-ray and regular follow-up. This subsidence was not associated with symptoms in all patients except one patient who had a marked loss of cervical lordosis and was symptomatic. It should be noted that adjacent segment disease was not reported in any of the cases throughout the whole follow-up period (Table 1).

\section{Discussion}

In the case of multi-level cervical disc degeneration with stenosis, the anterior approach remains more beneficial than the posterior approach in terms of directly addressing the sources of spinal cord compression, especially with a disturbed cervical spine sagittal profile, which is frequently found in such cases $[13,14]$. Although ACDF is a highly successful procedure for the treatment of degenerative cervical disc disease, the success rates were found to decline in multi-level discectomies as the number of levels increases [2].

Traditionally, anterior plating has been used to increase fusion rates and reduce subsidence and postoperative kyphosis. However, anterior plating may also be associated with potential disadvantages and complications, including prolonged operative time, screw loosening, screw pull-out, dysphagia, plate malposition, and accelerated adjacent segment degeneration $[3,15,16]$. The use of additional posterior instrumentation is effective but represents an additional operation with increased operative time and blood loss [17]. With the ongoing development of cage technology, stand-alone cervical cages have become more widely adopted by spine surgeons in single and multi-level ACDF to avoid the potential complications associated with the use of additional instrumentation [1]. However, research on this topic in relation to the application of four-level ACDF is insufficient. To our knowledge, the current series, including 130 patients, represents the largest study in the literature evaluating stand-alone cervical cages in cases undergoing four-level ACDF.
In this study, the indication of surgery was due to cervical myelopathy and/or radiculopathy. In all patients, an absolute spinal canal stenosis was observed in four levels based on the preoperative MRI examination. In some cases, radiological signs of myelopathy in the MRI without a clinical manifestation of myelopathy were observed.

\section{Clinical outcomes}

In this study, 130 patients experienced clinical improvement, manifested by the significant amelioration of NDI and VAS values at the final follow-up. This result is attributable to not only the successful procedure of ACDF since its initial description in 1955 but also the applicability of modern stand-alone cages for cervical fusion. Similar results have been achieved by most of the authors that focused on this topic. Li et al. [18] evaluated 27 patients undergoing stand-alone cage fusion for four-level cervical disc degeneration and reported a significant improvement in NDI from 25.5 to 12.6 after a mean of 36.9 months. Similarly, Wang et al. [3] examined 26 patients after standalone anchored cage fusion for four-level cervical spondylotic myelopathy and noted a significant improvement in NDI again from 26.6 to 14.2 after a minimum of 2 years of follow-up. Among the complications encountered in the current work, postoperative C5 palsy was noticed in three patients $(2.3 \%)$. The literature dealing with this specific item reported higher values. Krieg and Meyer [19] performed an extensive literature research via Medline and found an average value of $5 \%$ for postoperative C5 palsy, ranging from $0 \%$ to $30 \%$. Similarly, Wang et al. [20] underwent a meta-analysis for the incidence of postoperative C5 nerve palsy in the last decade and reported a value of $6.3 \%$. The higher values obtained in both studies might be due to the heterogeneity of the papers included in their search.

Another complication reported in this series is postoperative retropharyngeal hematoma, necessitating revisions in three patients (2.3\%). Song et al. [21] evaluated specifically this point after 785 anterior cervical fusion procedures. They reported an incidence of $1.15 \%$ for retropharyngeal hematoma. The higher value obtained in our work might be because we included exclusively fourlevel constructs, in contrast to the study of Song et al. [21] who examined every case undergoing fusion regardless of the number of levels operated. Besides the steep learning curve for this type of surgery, four-level constructs have a 
larger exposure area and, subsequently, a higher risk for bleeding. Furthermore, our threshold for revision in the case of retropharyngeal hematoma is low, with the result of no long-term complications in any of the cases with postoperative hematoma. In a single case, an intraspinal epidural hematoma presenting with a postoperative transient paraparesis was revised after MRI confirmation. After the revision, the patient did not have any residual deficits. Recurrent laryngeal nerve palsy did not represent a significant problem in the current series and occurred only transiently in one patient $(0.8 \%)$ despite the extent of the fusion. A similar value was reported by Tasiou et al. [22] who retrospectively evaluated 114 anterior cervical spine procedures and noticed an incidence of $0.9 \%$ for recurrent laryngeal nerve palsy. No iliac graft complications were observed in the postoperative period. Only 10 patients $(7.7 \%)$ had minimal discomfort in the site of the minimally invasive graft, and it was resolved after 10-30 days. This lower rate can be explained by using the cannulated drill through a minimally invasive approach without injury to the local tissues.

\section{Radiological outcomes}

Mechanical stability was a point of concern before applying this technique. In a comparative study conducted in 2012, four-level cage-assisted ACDF with and without additional posterior instrumentation was performed. The results of the study indicated that stand-alone intersomatic cage fusion is a good line of treatment for four-level cervical disc disease [17]. Thereafter, the four-level ACDF using stand-alone cages became a standard procedure in our institution. In the present study, cage subsidence necessitating revision due to kyphosis occurred in one patient $(0.8 \%)$. Furthermore, cervical lordosis was improved in this study from a mean of $5.5^{\circ}$ preoperatively to a final mean value of $10^{\circ}$, which was a statistically significant improvement. The findings obtained in this series have been confirmed by previous publications. Li et al. [18] compared the results of a four-level cage-assisted ACDF with and without anterior plating. In the group with stand-alone cages, including 27 patients, the mean cervical lordosis improved from $10.2^{\circ}$ to $17.1^{\circ}$. A similar improvement from $10.1^{\circ}$ to $19^{\circ}$ was reported when cervical plating was added. The difference between the two groups was not statistically significant.

Cho et al. [23] studied 180 cases of multi-level ACDF with three different fusion techniques. They found that stand-alone cages, as well as iliac graft with anterior cervical plating, are both satisfactory methods for interbody fusion. The complication rate was even lower in the standalone group. Recently, Wang et al. [3] evaluated four-level ACDF using stand-alone anchored cages and reported an improvement in the mean cervical lordosis from $8.7^{\circ}$ to $20^{\circ}$ postoperatively. However, a loss of cervical lordosis was observed over 24 months to reach a final mean value of $8.3^{\circ}$, being worse than the mean preoperative value. Although the direct postoperative mean cervical lordosis of $15^{\circ}$ could not also be maintained over the follow-up period in this study and decreased to reach $10^{\circ}$, the difference to the preoperative value remained significantly better. This better result compared with that of the study of Wang et al. [3] might be attributed to the design of the used cages and their application of artificial bone instead of autogenous iliac bone graft.

A further important aspect of ACDF is the achievement of bony fusion, which is of concern in the case of the multi-level disease. Although 19.23\% of our patients had at least one level with radiological non-solid bony fusion at the final follow-up, most of them were asymptomatic, and revision due to symptomatic pseudarthrosis was necessary for only two patients (1.5\%). A similar finding was reported by Li et al. [18], as $11.1 \%$ of their stand-alone four-level ACDF using polyetheretherketone (PEEK) cages showed pseudarthrosis, but none of them was symptomatic, necessitating revision. Wang et al. [20] reported a $23 \%$ rate of pseudarthrosis after performing stand-alone PEEK-anchored cages for four-level ACDF, but the revision was not needed in any of them. The values are similar to those of studies dealing with four-level ACDF adding instrumentation.

De la Garza-Ramos et al. [24] reported a pseudarthrosis rate of approximately $15.4 \%$ after performing instrumented ACDF for four-level disease. Li et al. [18] obtained a pseudarthrosis rate of $9.7 \%$ when adding a plate to the cages in their series. The high rate of radiological bony non-union after performing four-level ACDF can be easily explained by the increased number of fusion surfaces. However, in most cases and in most of the studies, this remains asymptomatic. Gerszten et al. [1] evaluated the results of multi-level ACDF using stand-alone cages and zero-profile instrumentation and concluded that both techniques are safe and effective, comparable with the reports that used plates [25]. The safety of stand-alone cages 
has been studied in a large cohort of patients as discussed in the literature [9].

In the present study, the high fusion rate and minimal cage sinking can be explained by several reasons. First, the cage design allowed the decortication of the non-weightbearing endplates without the cortical injury endplate. This technique reduces the rate of cage sinking. Second, the use of autologous bone graft may enhance fusion. Third, the use of surgical microscope allows a better decompression of the spinal canal and neuroforamen, resulting in good clinical improvement. Fourth, the use of titanium implants and titanium-coated implants has been proven to be more effective in the enhancement of fusion in previous studies [26]. Finally, anterior cervical plates were not used despite the long-segment fusion to reduce the complications associated with anterior cervical plating [9].

\section{Limitations}

The study had some limitations. One is the retrospective study design and absence of a control group for the comparison of the results obtained in the present study with the results of other techniques such as plate-supported fusion or the posterior approach. The results obtained in the present study may also be affected by the specific design of the used cages and the surgical techniques used. The endplates were only decorticated in the non-weight-bearing area of the cage, and the use of bone graft may have had a positive effect on the fusion rate.

\section{Conclusions}

The application of four-level ACDF using titanium cages in a stand-alone technique has been proven to be a safe and effective treatment method for degenerative disease. In a large cohort, a high rate of good clinical and radiological results with a minimum of 2 years of follow-up was achieved. Moreover, the anterior approach offers an alternative surgical solution for the four-level ACDF procedure in cases with segmental cervical stenosis.

\section{Conflict of Interest}

No potential conflict of interest relevant to this article was reported.

\section{Author Contributions}

Study design: MA, HB, MS; data collection: MA, HA; statistical analysis: MA; writing of the manuscript: MA; revision of the manuscript: $\mathrm{MA}, \mathrm{HA}, \mathrm{HB}$; reviewing the literature: MA; and critical reviewing of the Manuscript: MS.

\section{References}

1. Gerszten PC, Paschel E, Mashaly H, Sabry H, Jalalod'din H, Saoud K. Outcomes evaluation of zeroprofile devices compared to stand-alone PEEK cages for the treatment of three- and four-level cervical disc disease. Cureus 2016;8:e775.

2. Demircan MN, Kutlay AM, Colak A, et al. Multilevel cervical fusion without plates, screws or autogenous iliac crest bone graft. J Clin Neurosci 2007;14:723-8.

3. Wang B, Lu G, Kuang L. Anterior cervical discectomy and fusion with stand-alone anchored cages versus posterior laminectomy and fusion for four-level cervical spondylotic myelopathy: a retrospective study with 2-year follow-up. BMC Musculoskelet Disord 2018;19:216.

4. Cabraja M, Abbushi A, Koeppen D, Kroppenstedt S, Woiciechowsky C. Comparison between anterior and posterior decompression with instrumentation for cervica spondylotic myelopathy: sagittal alignment and clinical outcome. Neurosurg Focus 2010;28:E15.

5. Feng F, Ruan W, Liu Z, Li Y, Cai L. Anterior versus posterior approach for the treatment of cervical compressive myelopathy due to ossification of the posterior longitudinal ligament: a systematic review and meta-analysis. Int J Surg 2016;27:26-33.

6. Mobbs RJ, Rao P, Chandran NK. Anterior cervical discectomy and fusion: analysis of surgical outcome with and without plating. J Clin Neurosci 2007;14:639-42.

7. Resnick DK, Trost GR. Use of ventral plates for cervical arthrodesis. Neurosurgery 2007;60(1 Supp 1 1):S112-7.

8. Kim YS, Park JY, Moon BJ, Kim SD, Lee JK. Is stand alone PEEK cage the gold standard in multilevel anterior cervical discectomy and fusion $(\mathrm{ACDF})$ ?: results of a minimum 1-year follow up. J Clin Neurosci 2018;47:341-6.

9. Shousha M, Alhashash M, Allouch H, Boehm H. Reoperation rate after anterior cervical discectomy 
and fusion using standalone cages in degenerative disease: a study of 2,078 cases. Spine J 2019;19:200712.

10. Smith GW, Robinson RA. The treatment of certain cervical-spine disorders by anterior removal of the intervertebral disc and interbody fusion. J Bone Joint Surg Am 1958;40-A:607-24.

11. Bazaz R, Lee MJ, Yoo JU. Incidence of dysphagia after anterior cervical spine surgery: a prospective study. Spine (Phila Pa 1976) 2002;27:2453-8.

12. Chiang CJ, Kuo YJ, Chiang YF, Rau G, Tsuang YH. Anterior cervical fusion using a polyetheretherketone cage containing a bovine xenograftp: three to fiveyear follow-up. Spine (Phila Pa 1976) 2008;33:2524428.

13. Kato S, Ganau M, Fehlings MG. Surgical decisionmaking in degenerative cervical myelopathy: anterior versus posterior approach. J Clin Neurosci 2018;58:712.

14. Luo J, Cao K, Huang S, et al. Comparison of anterior approach versus posterior approach for the treatment of multilevel cervical spondylotic myelopathy. Eur Spine J 2015;24:1621-30.

15. Barbagallo GM, Romano D, Certo F, Milone P, Albanese V. Zero-P: a new zero-profile cage-plate device for single and multilevel ACDF: a single institution series with four years maximum follow-up and review of the literature on zero-profile devices. Eur Spine J 2013;22 Suppl 6(Suppl 6):S868-78.

16. Nambiar M, Phan K, Cunningham JE, Yang Y, Turner PL, Mobbs R. Locking stand-alone cages versus anterior plate constructs in single-level fusion for degenerative cervical disease: a systematic review and meta-analysis. Eur Spine J 2017;26:2258-66.

17. Shousha M, Ezzati A, Boehm H. Four-level anterior cervical discectomies and cage-augmented fusion with and without fixation. Eur Spine J 2012;21:25129.
18. Li Z, Wang H, Tang J, et al. Comparison of three reconstructive techniques in the surgical management of patients with four-level cervical spondylotic myelopathy. Spine (Phila Pa 1976) 2017;42:E575-83.

19. Krieg SM, Meyer B. C5 palsy after multi-segmental cervical decompression: how can it be avoided? Orthopade 2018;47:483-8.

20. Wang T, Wang H, Liu S, Ding WY. Incidence of C5 nerve root palsy after cervical surgery: a metaanalysis for last decade. Medicine (Baltimore) 2017;96:e8560.

21. Song KJ, Choi BW, Lee DH, Lim DJ, Oh SY, Kim SS. Acute airway obstruction due to postoperative retropharyngeal hematoma after anterior cervical fusion: a retrospective analysis. J Orthop Surg Res 2017;12:19.

22. Tasiou A, Giannis T, Brotis AG, et al. Anterior cervical spine surgery-associated complications in a retrospective case-control study. J Spine Surg 2017;3:44459.

23. Cho DY, Lee WY, Sheu PC. Treatment of multilevel cervical fusion with cages. Surg Neurol 2004;62:37885.

24. De la Garza-Ramos R, Xu R, Ramhmdani S, et al. Long-term clinical outcomes following 3- and 4-level anterior cervical discectomy and fusion. J Neurosurg Spine 2016;24:885-91.

25. Kreitz TM, Hollern DA, Padegimas EM, et al. Clinical outcomes after four-level anterior cervical discectomy and fusion. Global Spine J 2018;8:776-83.

26. Cuzzocrea F, Ivone A, Jannelli E, et al. PEEK versus metal cages in posterior lumbar interbody fusion: a clinical and radiological comparative study. Musculoskelet Surg 2019;103:237-41. 